\title{
HALL CONDUCTIVITY ESTIMATES FROM MAGNETOTELLURIC SOUNDING DATA
}

\section{V.V. Plotkin $\oplus^{凶}$, V.V. Potapov}

Trofimuk Institute of Petroleum Geology and Geophysics, Siberian Branch of the Russian Academy of Sciences, 3 Academician Koptyug Ave, Novosibirsk 630090, Russia

ABSTRACT. Many minerals have semiconductor properties. It is known that petroleum reservoir rocks permeated with hydrocarbon fluids can sometimes behave as semiconductors. In the Earth's magnetic field, the electrical conductivity of such materials becomes anisotropic, and the Hall effect is quite possible in rocks in natural conditions and detectable by magnetotelluric sounding. In the anisotropic medium, the field is subject to normal mode splitting, and its components show different attenuation coefficients and phase velocities. The modes differ due to polarization and rotation of the field vectors (clockwise in one mode, and counterclockwise in another). With account of the Hall effect, responses of the medium can be different when the medium is excited by a single normal wave. To detect the Hall effect in MTS surveys, we use the polarization analysis method and select the spectra of modes with right and left circular polarization. Special experiments were carried out to detect the contribution of the Hall effect during the MTS surveys. This article presents the first estimates of the Hall conductivity for the studied rocks.

KEYWORDS: magnetotelluric sounding (MTS); Hall effect; normal mode of anisotropic medium; Hall conductivity

FUNDING: The study was carried out with partial support from RFBR (project 17-05-00083) and FNI (project 03312019-0015 - Realistic theoretical models and software and methodological support for geoelectrics of heterogeneous geological media).

\section{RESEARCH ARTICLE}

Correspondence: Valery V. Plotkin, PlotkinVV@ipgg.sbras.ru
Received: January 2, 2019

Revised: April 6, 2020

Accepted: April 14, 2020

FOR CITATION: Plotkin V.V., Potapov V.V., 2020. Hall conductivity estimates from magnetotelluric sounding data. Geodynamics \& Tectonophysics 11 (4), 817-828. doi:10.5800/GT-2020-11-4-0509 


\title{
ОЦЕНКИ ХОЛЛОВСКОЙ ПРОВОДИМОСТИ ПО ДАННЫМ МАГНИТОТЕЛЛУРИЧЕСКОГО ЗОНДИРОВАНИЯ
}

\author{
В.В. Плоткин, В.В. Потапов
}

Институт нефтегазовой геологии и геофизики им. А.А. Трофимука СО РАН, 630090, Новосибирск, пр-т ак. Коптюга, 3, Россия

АНнотАЦИЯ. Многие минералы по своим свойствам являются полупроводниками. Замечено также, что вмещающие породы над залежами нефти и газа, пронизываемые углеводородными флюидами, иногда проявляют себя как полупроводники. Но так как электропроводность таких сред в магнитном поле Земли становится анизотропной, вполне возможны проявления эффекта Холла в горных породах в естественных условиях, например при магнитотеллурическом зондировании. В анизотропной среде поле расщепляется на составляющие, отличающиеся коэффициентами затухания и фазовой скоростью, - нормальные моды. Отличие мод связано с их поляризацией и направлением вращения вектора поля: в одной моде поле вращается по часовой стрелке, во второй - против. За счет эффекта Холла отклик среды может быть неодинаковым в случаях возбуждения среды лишь одной из нормальных волн. Для обнаружения влияния эффекта Холла при магнитотеллурическом зондировании мы используем метод поляризационного анализа с выделением спектров мод с правой и левой круговой поляризацией. Проведены специальные эксперименты для обнаружения вклада эффекта Холла при магнитотеллурическом зондировании, получены первые оценки холловской проводимости пород.

КЛЮЧЕВЫЕ СЛОВА: магнитотеллурическое зондирование; эффект Холла; нормальная мода анизотропной среды; холловская проводимость

ФИНАНСИРОВАНИЕ: Работа выполнена при частичной поддержке РФФИ (проект № 17-05-00083) и ФНИ (проект № 0331-2019-0015 «Реалистичные теоретические модели и программно-методическое обеспечение геоэлектрики гетерогенных геологических сред»).

\section{1. ВВЕДЕНИЕ}

Магнитотеллурическое зондирование (МТЗ) применяется для получения сведений о строении глубинных структур электропроводности земной коры и мантии (см., например [Pospeev, 2012; Novikov, Pospeeva, 2017; Moroz, Samoilova, 2018; Nikiforov et al., 2018; Rybin et al., 2019; Kelbert et al., 2012; Yang et al., 2015; Wunderman et al., 2018]). Глубинную структуру электропроводности определяют с помощью инверсии кривых МТЗ. Метод базируется на модели Тихонова-Каньяра (горизонтально-слоистая среда, возбуждаемая вертикально падающей плоской волной) [Semenov, 1985; Zhdanov, 1986; Berdichevsky, Dmitriev, 2009]. Однако из-за отличий геоэлектрического строения среды от этой модели часто возникают трудности при интерпретации магнитотеллурических кривых, преодолению которых посвящается множество работ [Wannamaker et al., 1984; Groom, Bahr, 1992; Singer, 1992; Caldwell et al., 2004; Kelbert et al., 2014; Avdeeva et al., 2015; Käufl et al., 2018; Kruglyakov, Kuvshinov, 2019].

Реальная геологическая среда сложна по строению, структуре, составу пород и механизмам электропроводности. Так, многие минералы по своим свойствам являются полупроводниками [Shuey, 1975]. Замечено также, что вмещающие породы над залежами нефти и газа, пронизываемые потоком углеводородных флюидов, ведут себя как полупроводники [Gololobov, Malevich, 2005].

Известно, что под действием магнитного поля в полупроводниках возможны проявления эффекта Холла.
В частности, это используется при лабораторных измерениях характеристик электропроводности (плотность и знак носителей заряда, их подвижность). При электромагнитных зондированиях вполне можно ожидать проявления эффекта Холла с подобными минералами, находящимися в горных породах в магнитном поле Земли в естественных условиях. Интерес к этому явлению как раз появился по результатам электромагнитных зондирований в районах ареалов углеводородов [Mogilatov, 2013].

Анализ лабораторных экспериментальных результатов измерения проводимости различных рудных минералов [Shuey, 1975] показывает, что в некоторых случаях вполне можно ожидать значений холловской проводимости $\sigma_{H} \leq 0.001 \mathrm{Cm} / \mathrm{м}$. Например, у галенита при средней проводимости образцов 1000 См/м, подвижности $\mu_{e} \sim 200 \mathrm{~cm}^{2} / \mathrm{B} \cdot \mathrm{c}, \mathrm{B} \sim 5 \cdot 10^{-5}$ Тл холловская проводимость $\sigma_{H}=0.001 \mathrm{Cm} /$ м. Несколько меньше холловская проводимость пирита, так как многие опубликованные значения подвижности электронов лежат в интервале 10-50 см ${ }^{2}$ В·с при такой же средней проводимости образцов. Пирит определяет электропроводность серного колчедана, и в зависимости от количества пирита ее величина колеблется в весьма широких пределах. Большой диапазон значений электропроводности горных пород (около двадцати порядков) обусловлен свойствами и составом входящих в них различных минералов. Это также касается и возможных значений холловской проводимости горных пород, поэтому использовать 
какие-либо достоверные ее значения станет возможным лишь после экспериментальных измерений $\sigma_{H}$ в естественных условиях. Ниже приводится описание и результаты первых специальных экспериментов по выявлению вклада эффекта Холла при МТЗ.

\section{2. КРАТКИЙ ОБЗОР ПРОБЛЕМЫ И МЕТОД АНАЛИЗА}

Обнаружить влияние постоянного магнитного поля Земли на электропроводность (эффект Холла) при магнитотеллурическом зондировании можно, если учесть вклад холловской проводимости $\sigma_{H}$ вводя ее в тензор электропроводности среды [Plotkin et al., 2019]. В анизотропной среде поле расщепляется на составляющие, отличающиеся коэффициентами затухания и фазовой скоростью, - нормальные моды. Отличие мод связано с их поляризацией и направлением вращения вектора поля, в одной моде поле вращается по часовой стрелке, во второй - против. Из физических соображений ясно, что за счет эффекта Холла отклик среды может быть неодинаковым в случаях возбуждения среды лишь одной из нормальных волн.

Анализ данных МТЗ показывает, что в общем случае направление вращения векторов магнитотеллурического (MT) поля (правая или левая поляризация) определяется характеристиками их частотного спектра. Хорошо известно, что поляризация эллиптическая и на разных частотах направление вращения вектора поля определяется разностью фаз спектральных составляющих горизонтальных компонент [Plotkin, Mogilatov, 2018]. Это можно объяснить соотношением амплитуд нормальных мод, представляющих МТ поле. Расчеты показали, что в средних широтах при слабом эффекте, когда $\sigma_{H} \ll \sigma$ (обычная электропроводность среды), коэффициенты поляризации мод в среде близки к значениям $\pm i$. Это означает, что в этих условиях нормальными являются моды с правой или левой круговой поляризацией. Амплитуды этих мод можно определить с помощью поляризационного анализа MT поля [Plotkin, 2018]. Для удобства приведем здесь его краткое описание. Разложение МТ поля при произвольной эллиптической поляризации на моды с правой и левой круговой поляризацией можно выполнить, используя следующее представление для реальной части горизонтальных компонент:

$$
\begin{aligned}
& \operatorname{Re}\left\{H_{x}(\omega) e^{i \omega t}\right\}=\operatorname{Re}\left\{A_{x}(\omega) e^{i\left(\varphi_{x}+\omega t\right)}\right\}= \\
& =A_{1} \cos \left(\varphi_{1}+\omega t\right)+A_{2} \cos \left(\varphi_{2}+\omega t\right), \\
& \operatorname{Re}\left\{H_{y}(\omega) e^{i \omega t}\right\}=\operatorname{Re}\left\{A_{y}(\omega) e^{i\left(\varphi_{y}+\omega t\right)}\right\}= \\
& =A_{1} \sin \left(\varphi_{1}+\omega t\right)-A_{2} \sin \left(\varphi_{7}+\omega t\right),
\end{aligned}
$$

где мода с индексом 1 характеризуется вращением горизонтальной компоненты магнитного поля против часовой стрелки, а мода с индексом 2 - по часовой. Амплитуды и фазы мод вычисляются по формулам поляризационного анализа:

$$
H_{1,2}(\omega)=A_{1,2} e^{\varphi_{1,2}}
$$

$$
\begin{gathered}
A_{1}^{2}=\frac{\left[A_{x}^{2}+A_{y}^{2}+2 A_{x} A_{y} \sin \left(\varphi_{x}-\varphi_{y}\right)\right]}{4}, \\
A_{2}^{2}=\frac{\left[A_{x}^{2}+A_{y}^{2}+2 A_{x} A_{y} \sin \left(\varphi_{y}-\varphi_{x}\right)\right]}{4}, \\
\operatorname{tg} \varphi_{1}=\frac{A_{x} \sin \varphi_{x}+A_{y} \cos \varphi_{y}}{A_{x} \cos \varphi_{x}-A_{y} \sin \varphi_{y}}, \operatorname{tg} \varphi_{2}=\frac{A_{x} \sin \varphi_{x}-A_{y} \cos \varphi_{y}}{A_{x} \cos \varphi_{x}+A_{y} \sin \varphi_{y}} .
\end{gathered}
$$

По аналогичным формулам осуществляется переход к модам и для горизонтальных компонент электрического поля. Затем для любой временной гармоники вместо стандартных импедансных соотношений между горизонтальными компонентами МТ-поля вводятся импедансные соотношения для мод с правой и левой круговой поляризацией:

$$
\begin{aligned}
& E_{1}(\omega)=Z_{11}(\omega) H_{1}(\omega)+Z_{12}(\omega) H_{2}(\omega), \\
& E_{2}(\omega)=Z_{21}(\omega) H_{1}(\omega)+Z_{22}(\omega) H_{2}(\omega),
\end{aligned}
$$

где мода с индексом 1 характеризуется вращением горизонтальной компоненты полей против часовой стрелки, а мода с индексом 2 - по часовой. Численное моделирование показывает, что различие импедансов мод $Z_{11}(\omega)$ и $Z_{22}(\omega)$ будет указывать на возможное присутствие эффекта Холла [Plotkin, Mogilatov, 2018]. Это различие можно также описать, переходя по обычным формулам от модулей импедансов к кривым кажущихся сопротивлений и к фазам введенных импедансов.

\section{3. РЕЗУЛЬТАТЫ СПЕЦИАЛЬНЫХ ЭКСПЕРИМЕНТОВ ПО ОБНАРУЖЕНИЮ ВКЛАДА ЭФФЕКТА ХОЛЛА ПРИ МТЗ И ИХ ОБСУЖДЕНИЕ}

Для обнаружения эффекта Холла в естественных условиях предпочтительно проводить исследования геологического разреза, максимально приближенного к горизонтально-слоистой среде. Исследование холловской проводимости в условиях сложно построенной среды значительно усложняет задачу, так как при наличии любых латеральных неоднородностей для того, чтобы заметить эффект, требуются увеличенные значения холловской проводимости.

После рекогносцировочных выездов было выбрано место (54 $51^{\prime}$ с.ш., $76^{\circ} 2^{\prime}$ в.д.) в Татарском районе Новосибирской области недалеко от пос. Орловка - южная часть Западно-Сибирской низменности в пределах Обь-Иртышской впадины. Местность характеризуется ровным спокойным рельефом (перепад высот 1 м на 60 км) и низкой структурно-геологической поверхностью. По априорным данным предыдущих геологических и геофизических исследований, район работ очень близок к горизонтально-слоистому разрезу. Ближайший поселок удален на расстояние 5 км, и вблизи нет никаких промышленных объектов и высоковольтных ЛЭП. Выбранный участок является хорошо изученным с геологической точки зрения, есть отчеты экспедиций (например, Барабинской геофизической экспедиции за 1948 г.), подтвержденные результатами бурения 
скважин (например, Татарской буровой партией за 1951 г.). На территории Татарского района проводились геофизические работы (гравиразведка, магниторазведка, электроразведка и сейсморазведка). В отчете Барабинской геофизической экспедиции «Геофизические исследования в центральной части Западно-Сибирской низменности» за 1948 г. отмечается, что электрические сопротивления всего комплекса горных пород изучаемого разреза в значительной степени дифференцированы. Подстилающими и опорными горизонтами в электрическом разрезе являются отложения палеозоя, характеризующиеся очень высоким, практически бесконечным, удельным электрическим сопротивлением (УЭС). По результатам обработки и интерпретации данных вертикального электрического зондирования (ВЭЗ) мощная толща рыхлых отложений мезокайнозоя представлена в своих низах толщей пород очень малого, но сравнительно постоянного УЭС (от 4 до 7 Ом·м), а в верхних горизонтах - менее мощными слоями горных пород с переменным УЭС (от 7 до 40 Ом·м). Толща горных пород с малым УЭС в низах мезокайнозоя (вероятнее всего, вся толща мела и юры) занимает в разрезе не менее 60-80 \% его мощности. Исходя из сводного разреза по результатам электроразведки и сейсморазведки, можно предположить, что среда является горизонтально-слоистой, отражающие горизонты в мезокайнозое, расположенные на глубинах 500 и 1000 м, залегают полого вплоть до глубины 2200 м. В итоге, по результатам изучения архивных данных ВЭЗ, сейсмических и гравиметрических данных по верхней части разреза до глубины 3300 м вблизи участка исследований можно сделать вывод о наличии нескольких пологих слоев, параллельных друг другу, без резких вертикальных границ и нарушений. Это позволяет с уверенностью говорить о горизонтально-слоистом характере среды.

Для проведения измерений методом МТЗ использовалась стандартная аппаратура MTU-5 фирмы Phoenix Geophysics (Канада), которая позволяла выполнять измерения на частотах от 300 до 0.0001 Гц. При проведении работ применялась типичная постановка станции MTU-5 на местности с 5-канальной регистрацией сигналов. Длина линий составляла 100 м. Первый эксперимент был проведен в июле 2018 г. с помощью одной станции. Регистрация сигналов длилась 40 ч. В 2019 г. в той же местности Татарского района были проведены повторные эксперименты на двух участках, время измерений составило 27 ч. На первом участке были поставлены две станции МТЗ: первая - в точке 2018 г. с осью $O X$ по направлению на магнитный север (точка 1-1), вторая на удалении 100 м с осью ОХ под углом $45^{\circ}$ к направлению на магнитный север (1-2). На втором участке на удалении 10 км сигналы были измерены одной станцией (вторая вышла из строя) с осью $О X$ под углом $5^{\circ}$ к направлению на магнитный север (2-1). В процессе измерений методом МТЗ в районе работ не наблюдалось гроз и других природных и техногенных явлений, ухудшающих шумовую обстановку для МТЗ.
Первоначальная оценка качества и обработка данных МТЗ осуществлялись программным обеспечением SSMT2000, поставляемым вместе с аппаратурой. Уровни сигналов во всех измерениях были нормальные, и корреляции во всем диапазоне (от 300 до 0.001 Гц) выше 0.5. Таким образом, качество первичных данных высокое. В качестве примера на рис. 1 приведены кривые кажущегося сопротивления и фазы для точки 1-1 за 2019 г. На полярных диаграммах всех точек видно, что для частот десятые доли Гц и ниже диаграммы теряют круглую форму, что говорит о том, что нарушается горизонтальное строение среды, но даже и на меньших частотах овалы на диаграммах не сильно вытянуты, что говорит лишь о небольших отклонениях от горизонтальной слоистости. Для частот выше десятых долей Гц диаграммы круглые. Сравнивая кривые кажущихся сопротивлений всех точек, можно сказать, что они идентичны, не расходятся для разных поляризаций, что еще раз говорит о горизонтальной слоистости среды и ее малом изменении при перемещении от точки к точке. После интерпретации получены близкие модели среды для каждой из точек (с учетом эквивалентности), представленных в табл. 1.

Для выявления вклада эффекта Холла экспериментальные данные были обработаны предложенным методом поляризационного анализа. Как уже указано, при этом импедансные соотношения между горизонтальными компонентами МТ поля заменяем линейными связями между амплитудами независимых мод с правой и левой круговой поляризацией. По своей сути модовые импедансные соотношения аналогичны стандартным линейным связям между ортогональными компонентами МТ поля, поэтому методика определения стандартных импедансов в SSMT2000 вполне применима для нахождения их модовых аналогов. Отличие лишь в том, что предварительно по формулам поляризационного анализа производим переход от амплитуд временного спектра горизонтальных компонент МТ поля к амплитудам мод с правой и левой круговой поляризацией. Поскольку присутствует элемент новизны, было решено сравнить результаты обработки по новой методике данных пунктов 1-1 и 1-2 (с развернутой на $45^{\circ}$ установкой) 2019 г. Для повышения

Таблица 1. Модели среды по данным 2019 г.

Table 1. Models of the medium based on the data obtained in 2019

\begin{tabular}{lrrrrrr}
\hline \multirow{2}{*}{ № } & \multicolumn{2}{c}{ Точка 1-1 } & \multicolumn{2}{c}{ Точка 1-2 } & \multicolumn{2}{c}{ Точка 2-1 } \\
\cline { 2 - 7 } & H, км & $\rho$, Ом·м & $H$, км & $\rho$, Ом·м & $H$, км & $\rho$, Ом·м \\
\hline 1 & 0.04 & 5.35 & 0.07 & 5.88 & 0.15 & 8.32 \\
2 & 0.28 & 8.31 & 0.24 & 8.48 & 0.77 & 6.04 \\
3 & 1.38 & 3.99 & 1.21 & 3.41 & 4.32 & 14.60 \\
4 & 14.94 & 97.00 & 59.93 & 201.79 & 75.00 & 400.00 \\
5 & & 158.24 & & 94.59 & & 66.79 \\
\hline
\end{tabular}


достоверности получаемых по новой методике результатов мы сравниваем их с аналогичными данными пункта в том же месте в 2018 г. Чтобы выяснить, влияют ли отклонения от горизонтальной слоистости среды, были обработаны независимые данные удаленного пункта 2-1 2019 г.

Для сравнения и общего представления на рис. 2 даны стандартные и модовые кривые МТЗ, определен- ные по экспериментальным данным за два года во всех пунктах (учитывались лишь данные с корреляцией более 0.9). Кривые в соответствующих вариантах в пределах экспериментальных погрешностей совпадают. Следует отметить наличие окон на периодах 5-10 с в поведении всех полученных кривых, когда поле первичного источника на этих периодах при регистрации данных отсутствовало.

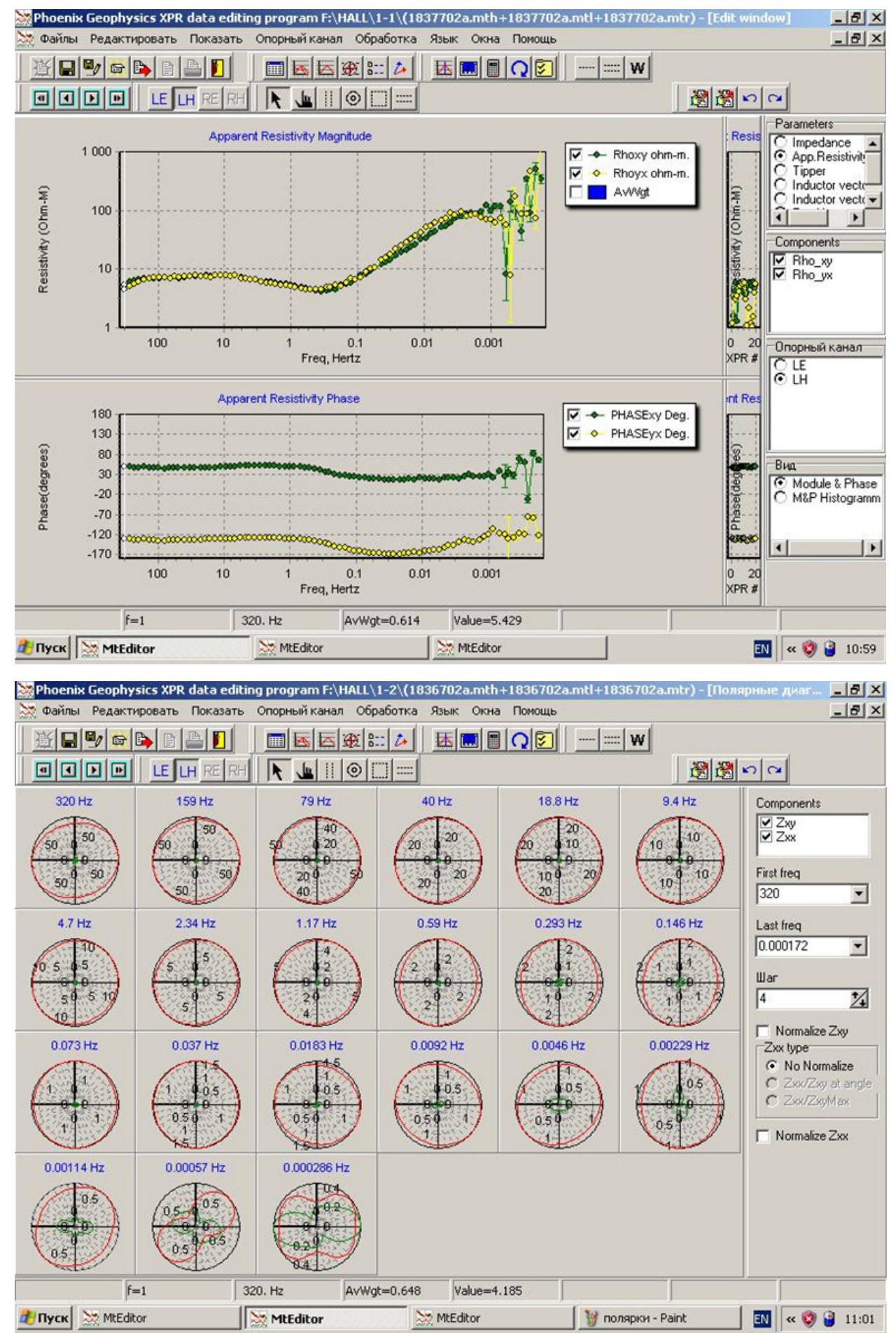

Рис. 1. Кривые кажущегося сопротивления и фазы, полярные диаграммы для точки 1-1 за 2019 г.

Fig. 1. Apparent resistivity and phase curves, and polar diagrams (data on point 1-1, 2019). 

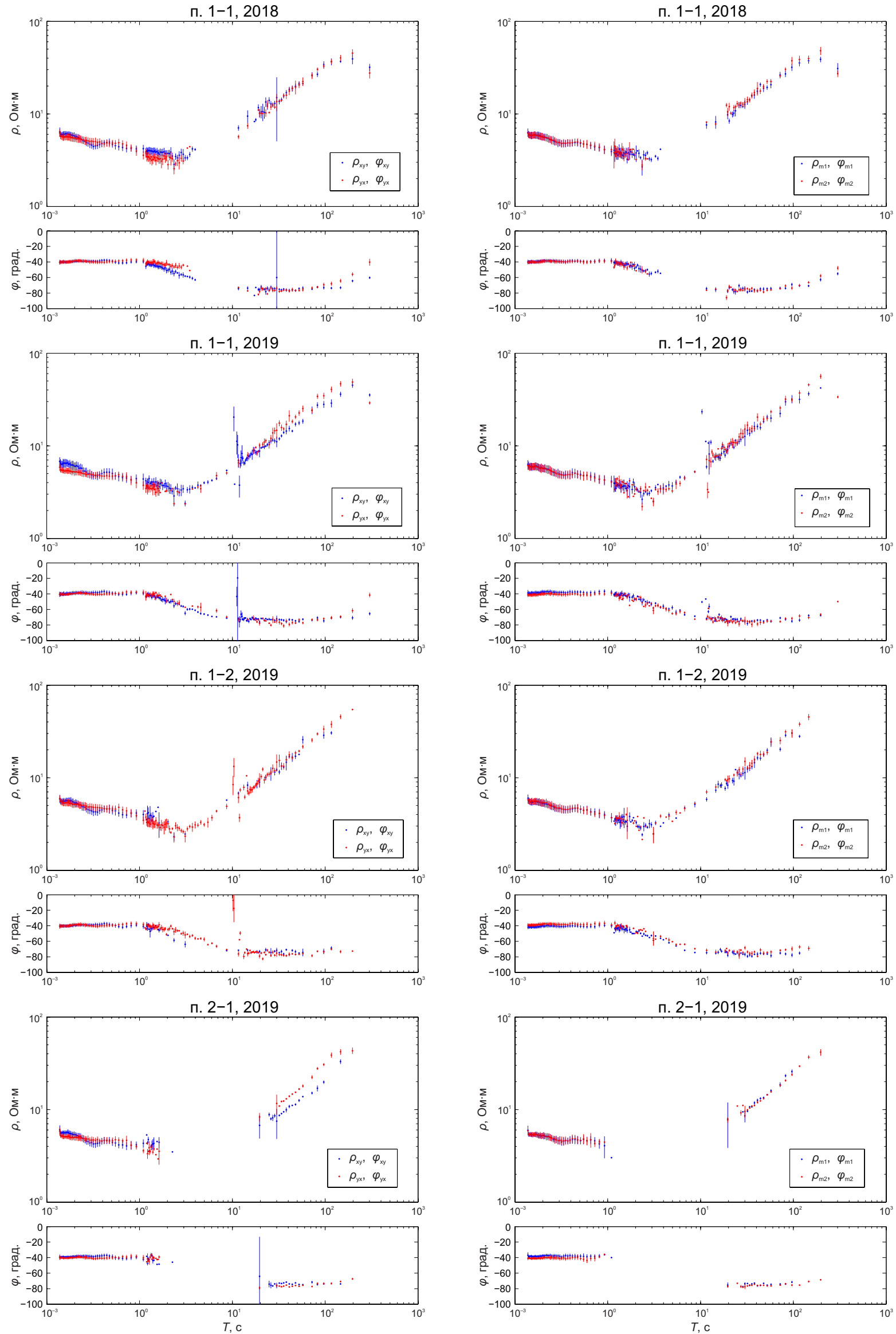

Рис. 2. Стандартные (слева) и модовые (справа) кривые МТЗ по данным 2018 и 2019 гг.

Fig. 2. Standard and mode MTS curves (left and right, respectively) (data obtained in 2018 and 2019). 
Меньше всего подобных пропусков было в пунктах 1-1 и 1-2 2019 г. Это вселяет надежду на то, что данные на таких периодах все же могут быть получены при более длительных наблюдениях. Однако пока возможность получения надежных оценок величины холловской проводимости по данным всего диапазона рассматриваемых временных периодов отсутствует.

В связи с этим далее рассматривался вариант получения нескольких оценок величины холловской проводимости по отдельным участкам кривых в двух диапазонах временных периодов. Предполагалось уточнить приведенные модели среды, учитывая в них возможный вклад холловской проводимости.

Полученные по данным п. 1-12019 г. зависимости модулей стандартных импедансов от периода и полярные диаграммы для двух выбранных диапазонов периодов показаны на рис. 3. На периодах меньше 1 с полярные диаграммы главного импеданса близки к круговым. Это подтверждает высказанное нами предположение, что на коротких временных периодах выбранный участок вполне соответствует горизонтально-слоистой среде, поэтому оценки холловской проводимости на коротких периодах проводились алгоритмом, разработанным для такой среды [Plotkin, 2017].

Оптимизация модели горизонтально-слоистой среды осуществлялась минимизацией функционала $\Phi$ суммы всех невязок стандартных и модовых кривых:

$\Phi=\frac{1}{4 K} \sum_{k=1}^{K}\left(\left|\frac{\rho_{m 1}-\rho_{m 1}^{0}}{\rho_{m 1}^{0}}\right|_{k}^{2}+\left|\frac{\rho_{m 2}-\rho_{m 2}^{0}}{\rho_{m 2}^{0}}\right|_{k}^{2}+\left|\frac{\rho_{x y}-\rho_{x y}^{0}}{\rho_{x y}^{0}}\right|_{k}^{2}+\left|\frac{\rho_{y x}-\rho_{y x}^{0}}{\rho_{y x}^{0}}\right|_{k}^{2}\right.$,

где $K$ - количество учитываемых периодов, $\rho_{m 1,2}, \rho_{m 1,2}^{0}-$ соответственно модельные и экспериментальные модовые кажущиеся сопротивления и $\rho_{x y, y x}, \rho_{x y, y x}^{0}-$ соответственно модельные и экспериментальные стандартные кажущиеся сопротивления.

Как видно из рис. 2, модовые кривые на коротких периодах практически совпадают. Это означает малость вклада или отсутствие холловской проводимости $\sigma_{H}$ на глубинах, соответствующих учитываемым периодам. Результаты оптимизации модели среды в диапазоне периодов 0.1-10.0 с отражены на рис. 4 для двух наборов данных пункта 1-1 2019 г. (первый - рис. 4, а, в, д и второй - рис. 4, б, г, е). В ходе оптимизации по данным показанных периодов переменными являлись характеристики глубинного разреза (мощности четырех слоев, электропроводности их и подстилающей среды), а также холловская проводимость $\sigma_{H}$ (она принималась одинаковой во всех слоях среды). На рис. 4, а, б, показаны модели горизонтально-слоистой среды (сплошная линия - модель, полученная с помощью палеток по кривой $\rho_{x y}^{0}$ на рис. 1 , пунктир - модели, полученные по результатам их оптимизации). На рис. 4 , в, г, отражены стандартные кажущиеся сопротивления $\rho_{x y, y x}$ в анализируемых диапазонах временных периодов (кажущиеся сопротивления представлены в линейном масштабе по оси абсцисс в узком диапазоне 2-7 Ом·м). На рис. 4 , д, е, аналогичным методом отражены модовые кажущиеся сопротивления $\rho_{m 1,2}$. Погрешности экспериментального определения кажущихся сопротивлений в изучаемом диапазоне 1.0-1.5 Ом·м. Для получения непрерывных кривых кажущихся сопротивлений нами применялась аппроксимация их зависимостей от периода полиномами.

Оптимизация модели среды выполнялась методом Нелдера-Мида по сглаженным кривым (отметим, что минимизация функционала $\Phi$ осуществлялась без учета логарифмического характера распределения кажущегося сопротивления из-за узкого диапазона взятых кажущихся сопротивлений).

Как видно на рис. 4, в, полученные для первого набора данных сглаженные экспериментальные кривые $\rho_{x y}^{0}$ и $\rho_{y x}^{0}$ не совпадают, отличаясь в рассматриваемом диапазоне периодов по величине до $\sim 0.5$ Ом·м. Конечно, это не имеет место для горизонтально-слоистой среды, и взятые для такой среды алгоритмы оценок холловской проводимости при оптимизации ее модели не смогли привести к точному совпадению экспериментальных и модельных кривых, поэтому дополнительно была проведена оптимизация по данным более короткого диапазона периодов, результаты которой отражены на рис. 4, б, г, е. В этом случае экспериментальные кривые $\rho_{x y}^{0}$ и $\rho_{y x}^{0}$ практически совпали, а холловская проводимость оказалась близкой к нулю. Несмотря на меньшие значения $\Phi$, полного совпадения модельных и экспериментальных кривых достигнуть не удается (найденные значения минимумов $\Phi$ и соответствующих его слагаемых показаны для каждого случая).

Модели среды на рис. 4, а, б, полученные по результатам оптимизации в разных диапазонах периодов, заметно отличаются лишь на небольших глубинах - менее 400 м. Ниже этого горизонта модели согласуются друг с другом в большей степени, что служит обоснованием их достоверности на таких глубинах. В то же время оценки холловской проводимости отличаются существенно. Понятно, что для более надежных оценок слабого вклада эффекта Холла необходимо иметь и более точную модель фоновой среды. Полученные результаты показывают лишь верхний порог возможных значений холловской проводимости. Отметим, что результаты оценок холловской проводимости по данным 2018 [Plotkin, Mogilatov, 2018] и 2019 гг. на периодах 0.1-10.0 с примерно совпадают. Можно утверждать, что ее предполагаемые значения не превышают $\sim 10^{-3} \mathrm{Cm} /$ м. Для повышения надежности оценок $\sigma_{H}$ следует уменьшить погрешности определения кажущихся сопротивлений накоплением данных в более продолжительных сеансах измерений.

Результаты оптимизации модели среды на периодах 10-100 с отражены на рис. 5. Полярные диаграммы на рис. 3 для этого диапазона периодов соответствуют модели 2D-среды. Кривые $\rho_{x y}^{0}$ и $\rho_{y x}^{0}$ на таких периодах по данным 2019 г. в п. 1-1 и 2-1 (см. рис. 2) не совпадают, поэтому для оптимизации модели среды на увеличенных глубинах использовался алгоритм, разработанный на базе метода Треффца [Plotkin et al., 2019]. Он позволяет 
определять кривые МТЗ для трехмерно-неоднородной среды с холловской проводимостью. С учетом полярных диаграмм, приведенных на рис. 3, нами использовались модели 2D-среды с изменениями ее параметров по латерали вдоль оси $O X$ на глубинах более 500 м.

Однако погрешности экспериментального определения кажущихся сопротивлений в этом диапазоне возросли до $\sim 2$ Ом·м. Для большей надежности и накопления данных на периодах 10-100 с были объединены результаты определений кажущихся сопротивлений в п. 1-1 за 2018 и 2019 гг. На рис. 5, а, б, в, показаны модели среды (сплошная - модель горизонтально-слоистой среды, полученная с помощью палеток по кривой $\rho_{x y}^{0}$ на рис. 1, пунктир - модели 2D-среды, полученные по результатам их оптимизации). На рис. 5, г, д, е, отражены стандартные кажущиеся сопротивления $\rho_{x y, y x}$ в анализируемых диапазонах временных периодов (кажущиеся сопротивления представлены в линейном масштабе
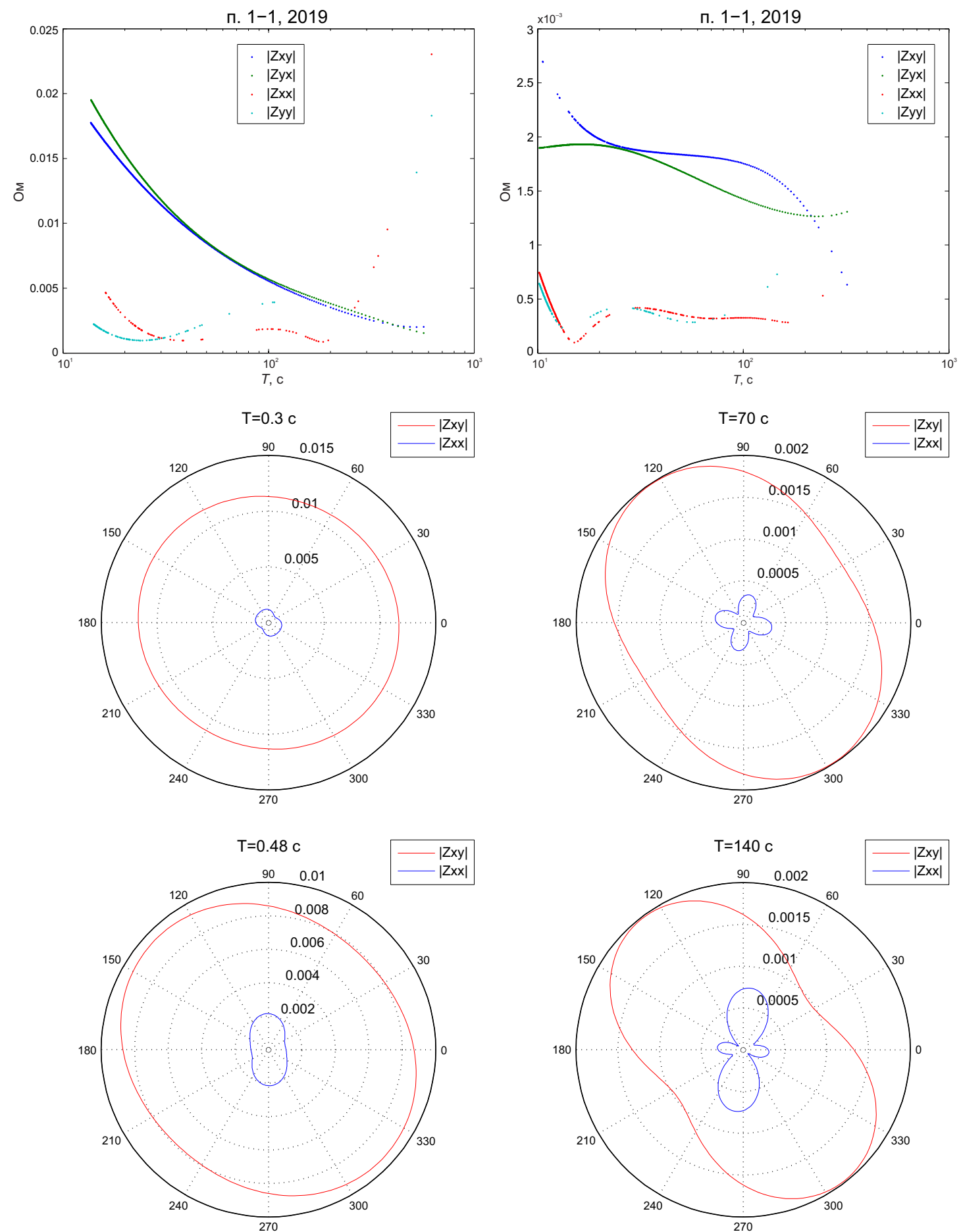

Рис. 3. Модули импедансов в двух диапазонах временных периодов и полярные диаграммы по данным п. 1-1 2019 г.

Fig. 3. Impedance modules in two time ranges and polar diagrams (data on point 1-1, 2019). 

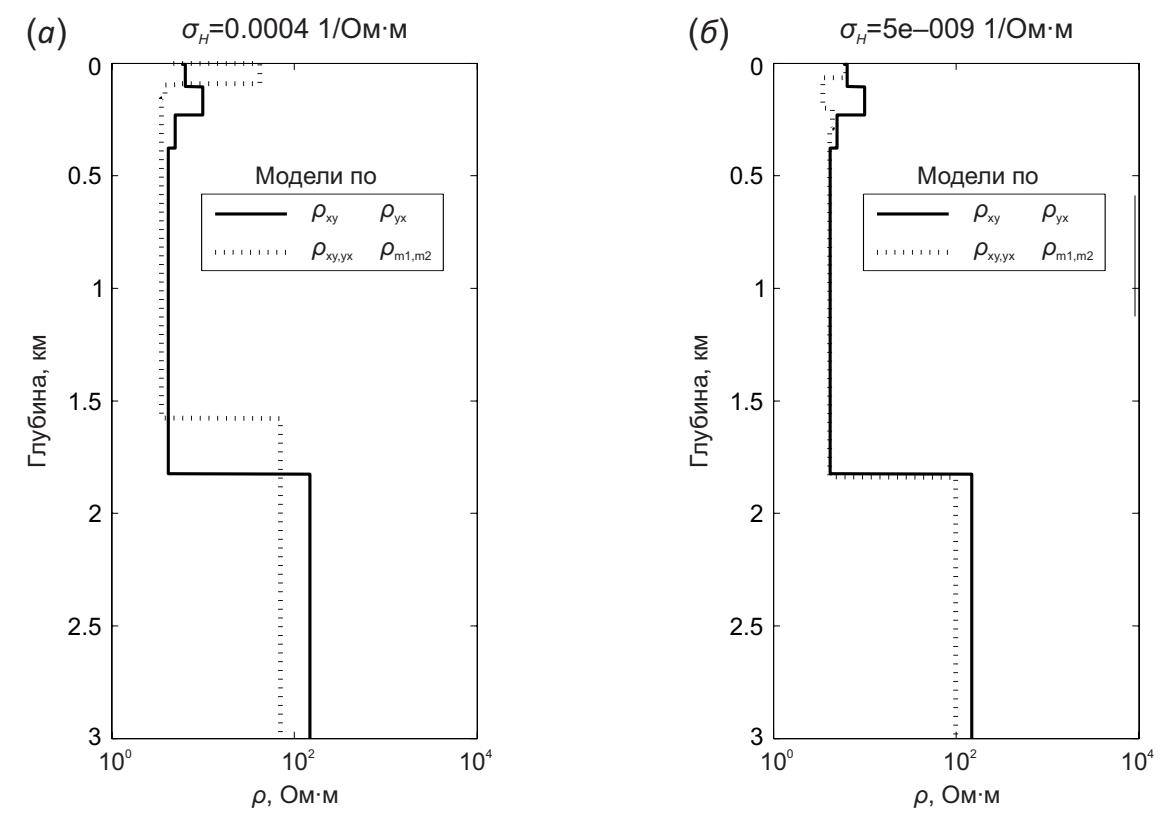

(8)

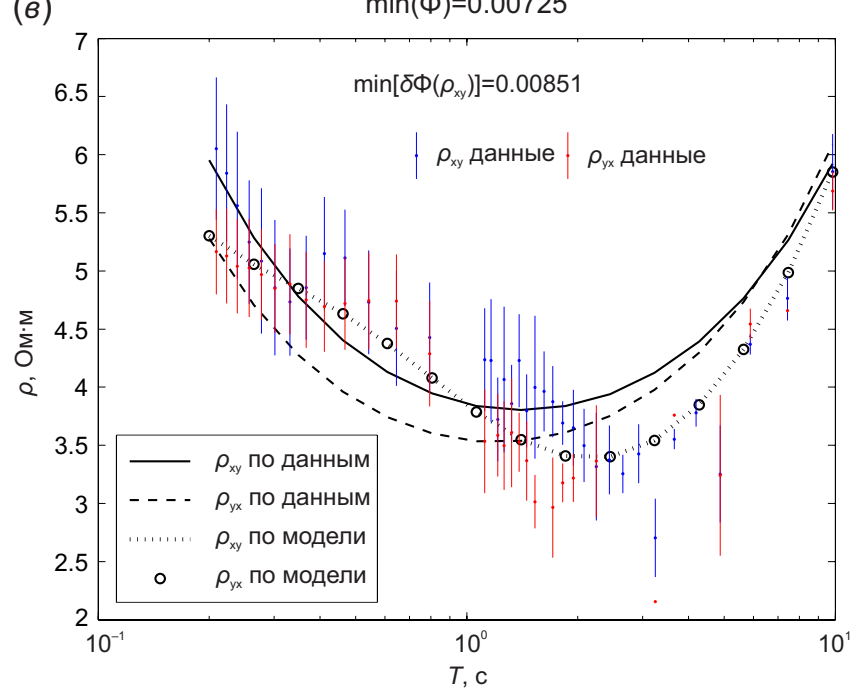

(d)

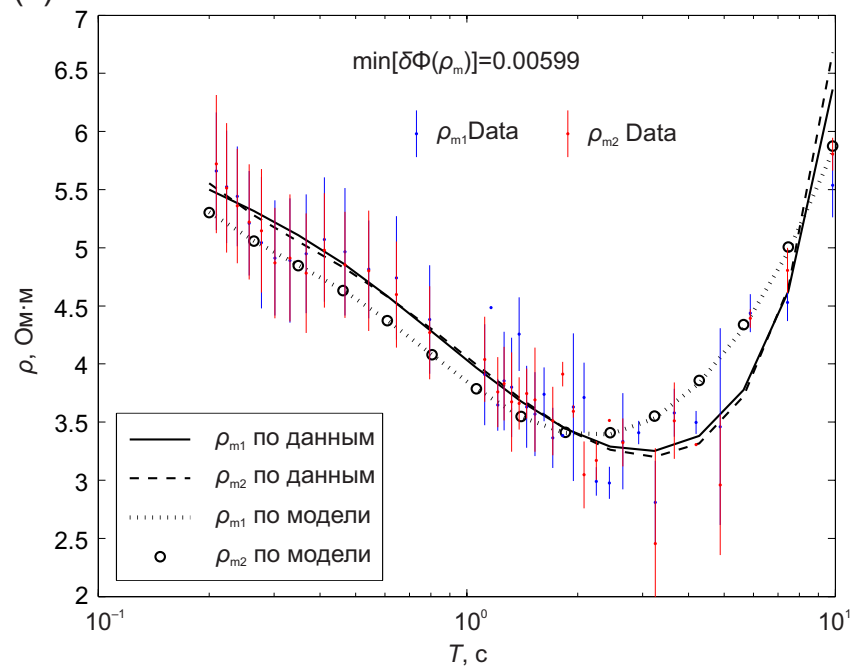

(2)

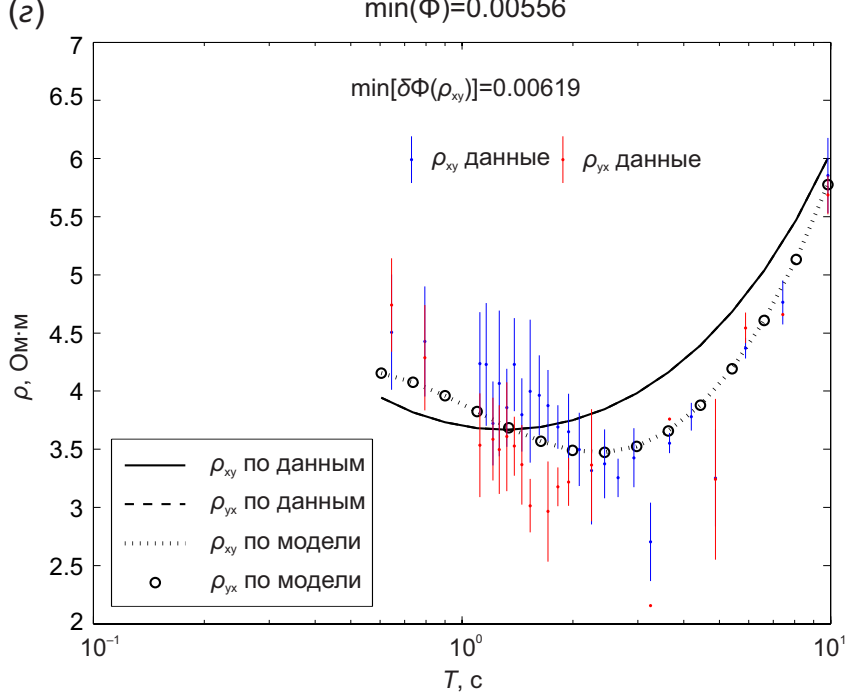

(e)

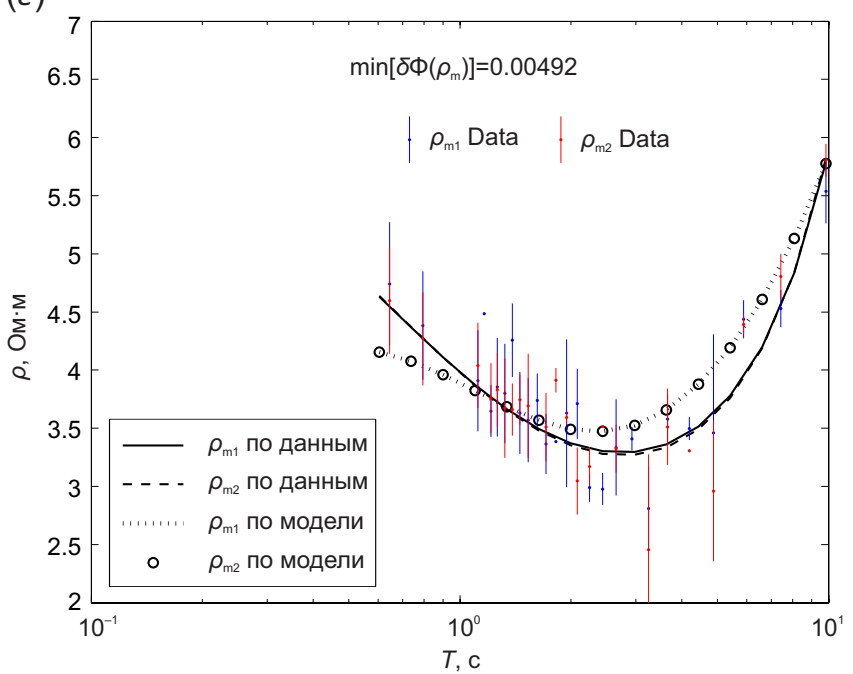

Рис. 4. Результаты оптимизации модели горизонтально-слоистой среды с учетом эффекта Холла для двух выборок данных на периодах 0.1-10.0 с ( $a$, б - модели среды, 6,2 - стандартные и $\partial, e$ - модовые кривые кажущегося сопротивления).

Fig. 4. Optimization of horizontal-layered medium models taking into account the Hall effect for two data sets in 0.1-10.0 s time intervals ( $a, 6$ - models; apparent resistivity curves: 8,2 -standard, $\partial, e$-mode). 
по оси абсцисс в узком диапазоне 2-7 Ом·м). На рис. 5, ж, 3, и, аналогичным методом отражены модовые кажущиеся сопротивления $\rho_{m 1,2}$.

На графиках рис. 5, а, г, ж, отражены итоги оптимизации для более широкого диапазона периодов в сравнении с остальными графиками. При оптимизации переменными считались электропроводности в четвертом сверху слое и холловская проводимость $\left(\sigma_{H}\right.$ принималась одинаковой во всех слоях среды). Холловская проводимость оказалась равной 0.0008 См/м (достигнутые значения $\Phi$ и соответствующих слагаемых приведены).

Для более узкого диапазона периодов (рис. 5, б, д, 3) удалось получить лучшее соответствие между модельными и экспериментальными кривыми как стандартных, так и модовых кривых (уменьшенные значения минимума $\Phi$ и слагаемых). При этом холловская проводимость оказалась равной -0.035 См/м, хотя в предыдущем случае ее значение существенно меньше $\sim 10^{-3}$ См/м и другого знака.

Чтобы снять это противоречие, в число варьируемых параметров модели 2D-среды в последнем варианте было добавлено еще две переменных - толщина четвертого слоя и электропроводность подстилающего слоя. Результаты оптимизации такой модели представлены на графиках рис. 5, в, е, и. Соответствие модельных и экспериментальных кривых стало лучше (см. достигнутые значения $\Phi$ и слагаемых). Величина холловской проводимости $-0.0004 \mathrm{Cm} / \mathrm{M}$.

Как видно, модели 2D-среды на рис. 5, а, б, в, больше отличаются друг от друга в сравнении с упомянутыми изменениями моделей на рис. 4, а, б. Это объясняется возрастанием погрешностей экспериментального

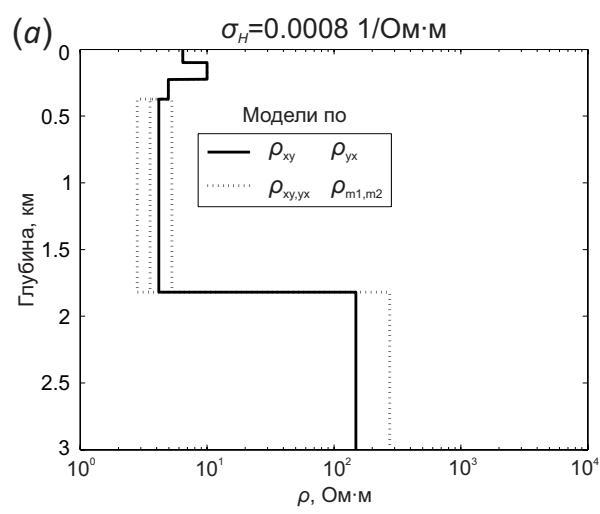

(2)

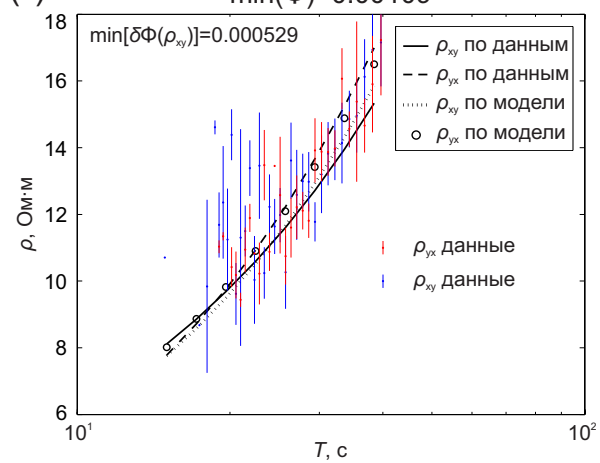

(ж)

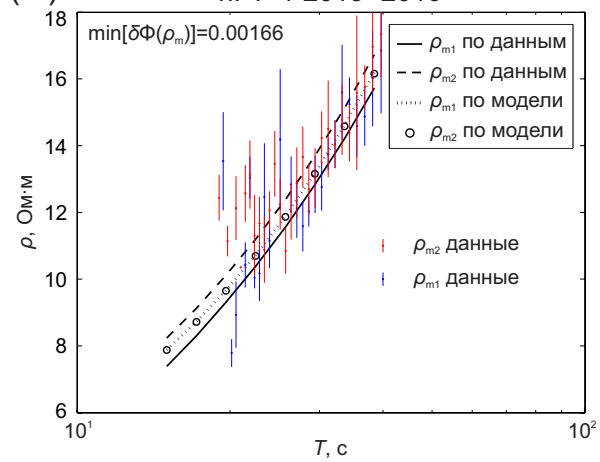

(б)

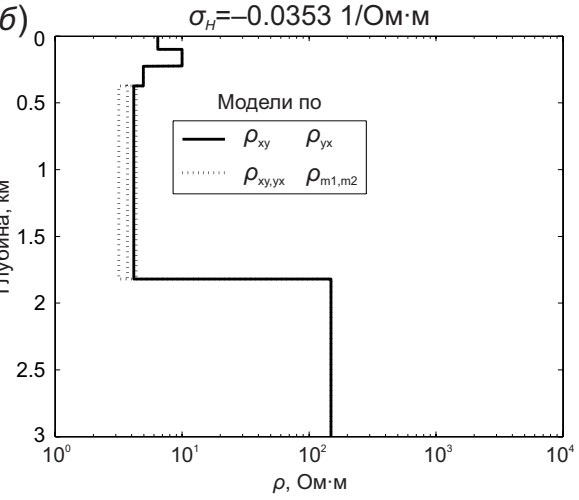

(d)

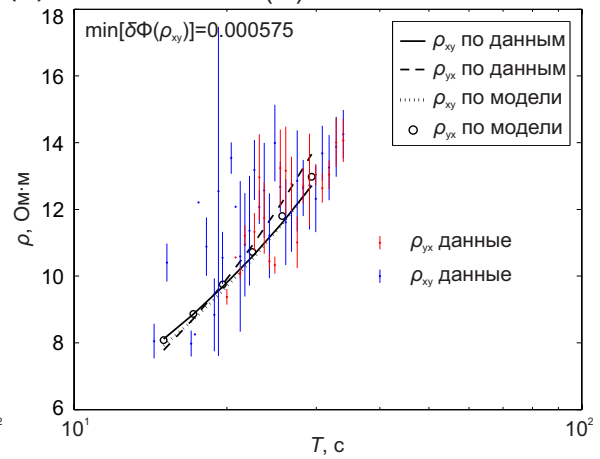

(3)

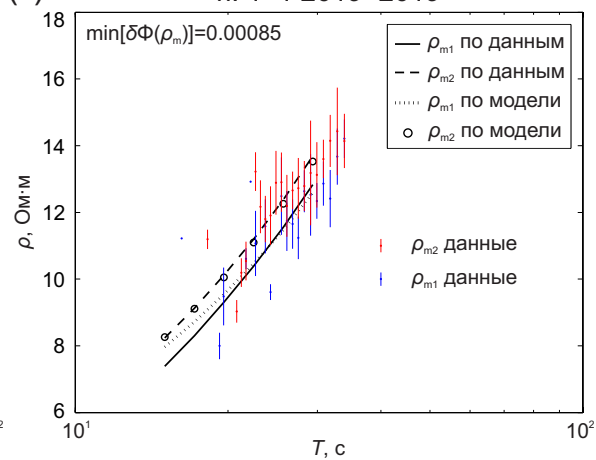

(8)

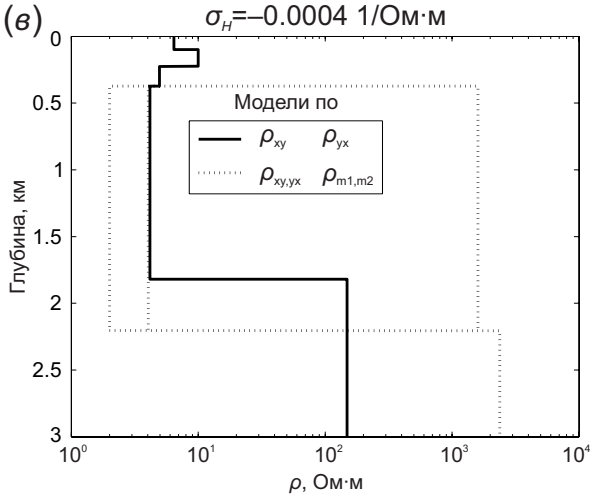

(e)

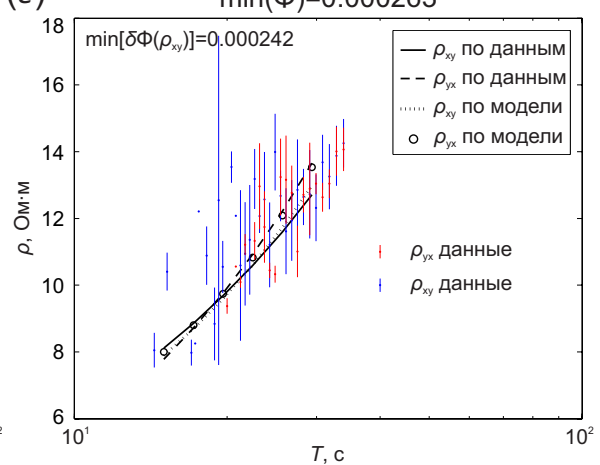

(u)

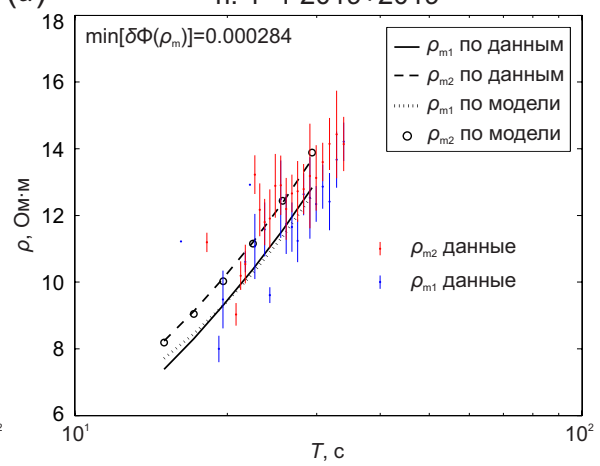

Рис. 5. Результаты оптимизации модели латерально неоднородной 2D-среды сучетом эффекта Холла для трех вариантов данных на периодах 10-100 с ( $a, 6,8$ - модели среды, 2, , $e$ - стандартные и ж, 3, и - модовые кривые кажущегося сопротивления).

Fig. 5. Optimization of laterally non-uniform 2D medium models taking into account the Hall effect for three data variants in 10-100 $\mathrm{s}$ time intervals ( $a, \sigma, \varepsilon$ - models; apparent resistivity curves: $2, \partial, e$-standard, $\varkappa_{,}, 3, u$ - mode). 
определения кажущихся сопротивлений в диапазоне 10-100 с. Больший разброс получается и по значениям холловской проводимости (вплоть до изменения ее знака). Тем не менее можно утверждать, что верхний порог предполагаемых значений $\sigma_{H}$ по модулю на периодах 10-100 с также не превышает $\sim 10^{-3}$ См/м.

\section{4. ЗАКЛЮЧЕНИЕ}

Для обнаружения вклада эффекта Холла в естественных условиях были проведены специальные эксперименты в Татарском районе Новосибирской области, недалеко от пос. Орловка (5451' с.ш., 76²' в.д.). Это место выбрано исходя из максимальной близости разреза к горизонтально-слоистой среде и сложности определения вклада холловской проводимости в условиях сложно построенной среды. Для проведения измерений методом МТЗ использовалась стандартная аппаратура MTU-5 фирмы Phoenix Geophysics (Канада).

Поставленная задача решена с помощью определения амплитуд мод с правой и левой круговой поляризацией и анализом модовых соотношений между найденными амплитудами вместо стандартных импедансных. Это связано с тем, что за счет эффекта Холла отклик среды может быть неодинаковым в случаях возбуждения среды лишь одной из нормальных волн. Различие модовых импедансов указывает на возможное присутствие эффекта Холла. Это различие можно также описать, переходя по обычным формулам от модулей импедансов к кривым кажущихся сопротивлений и к фазам введенных импедансов.

После обработки экспериментальных данных за два года получены стандартные и модовые кривые МТЗ в выбранном месте в нескольких пунктах (для проверки обработки данных по новой методике сравнивались результаты нескольких пунктов). Кривые в соответствующих вариантах в пределах экспериментальных погрешностей совпали. Отмечено наличие окон на периодах 5-10 с в поведении полученных кривых, поэтому пока возможность получить оценки холловской проводимости по данным всего диапазона учитываемых временных периодов отсутствует. Проведены оценки холловской проводимости по отдельным участкам кривых в двух диапазонах временных периодов. Поскольку для более надежных оценок слабого вклада эффекта Холла необходимо иметь точную модель фоновой среды, нам удалось установить лишь верхний порог возможных значений холловской проводимости. По полученным оценкам ее значения не превышают $\sim 10^{-3} \mathrm{Cm} / \mathrm{M}$, поэтому необходимы дальнейшие эксперименты с продолжительными измерениями для накопления более точных данных о кажущихся сопротивлениях геологической среды.

Следует подчеркнуть, что в случае успеха определение холловской проводимости геологической среды позволяет выявлять дополнительные характеристики ее электропроводности - плотность, знак и подвижность носителей заряда, а также тип проводимости (ионная или электронная).

\section{5. БЛАГОДАРНОСТИ}

Работа выполнена при частичной поддержке РФФИ (проект № 17-05-00083) и ФНИ (проект № 0331-20190015 - «Реалистичные теоретические модели и программно-методическое обеспечение геоэлектрики гетерогенных геологических сред»).

\section{6. ЛИТЕРАTУРА / REFERENCES}

Avdeeva A., Moorkamp M., Avdeev D., Jegen M., Miensopust M., 2015. Three-Dimensional Inversion of Magnetotelluric Impedance Tensor Data and Full Distortion Matrix. Geophysical Journal International 202 (1), 464-481. https://doi.org/10.1093/gji/ggv144.

Berdichevsky M.N., Dmitriev V.I., 2009. Models and Methods of Magnetotellurics. Nauchny Mir, Moscow, 668 p. (in Russian) [Бердичевский М.Н., Дмитриев В.И. Модели и методы магнитотеллурики. М.: Научный мир, 2009. 668 c.].

Caldwell T.G., Bibby H.M., Brown C., 2004. The Magnetotelluric Phase Tensor. Geophysical Journal International 158 (2), 457-469. https://doi.org/10.1111/j.1365-246X. 2004.02281.x.

Gololobov D.V., Malevich I.Yu., 2005. Physical and Electrochemical Processes in the Medium over a Carbon Deposit. Doklady BGUIR 1, 22-27 (in Russian) [Гололобов Д.В., Малевич И.Ю. Физические и электрохимические процессы в среде над залежью углеродов // Доклады БГУИР. 2005. № 1. C. 22-27].

Groom R.W., Bahr K., 1992. Corrections for near Surface Effects: Decomposition of the Magnetotelluric Impedance Tensor and Scaling Corrections for Regional Resistivities: A Tutorial. Survey Geophysics 13, 341-379.

Käufl J.S., Grayver A.V., Kuvshinov A.V., 2018. Topographic Distortions of Magnetotelluric Transfer Functions: A HighResolution 3-D Modelling Study Using Real Elevation Data. Geophysical Journal International 215 (3), 1943-1961. https://doi.org/10.1093/gji/ggy375.

Kelbert A., Egbert G.D., deGroot Hedlin C., 2012. Crust and Upper Mantle Electrical Conductivity beneath the Yellowstone Hotspot Track. Geology 40 (5), 447-450. https: / doi.org/10.1130/G32655.1.

Kelbert A., Meqbel N., Egbert G.D., Tandon K., 2014. ModEM: A Modular System for Inversion of Electromagnetic Geophysical Data. Computers \& Geosciences 66, 40-53. https: // doi.org/10.1016/j.cageo.2014.01.010.

Kruglyakov M., Kuvshinov A., 2019. 3-D Inversion of MT Impedances and Inter-Site Tensors, Individually and Jointly. New Lessons Learnt. Earth, Planets and Space 71 (4). https:// doi.org/10.1186/s40623-018-0972-8.

Mogilatov V.S., 2013. On the Influence of the Geomagnetic Field on the Process of Establishing Currents in the Ground. Geophysics 4, 70-75 (in Russian) [Могилатов B.C. О влиянии геомагнитного поля на процесс установления токов в земле // Геофизика. 2013. № 4. С. 70-75].

Moroz Yu.F., Samoilova O.M., 2018. On the Geoelectrical Difference between the Kuril-Kamchatka and Bering Sea Segments of the Pacific Transition Zone. Geodynamics \& Tectonophysics 9 (2), 489-501 (in Russian) [Мороз Ю.Ф., 
Самойлова О.М. О геоэлектрическом различии Курило-Камчатского и Беринговоморского сегментов Тихоокеанской зоны перехода // Геодинамика и тектонофизика. 2018. Т. 9. № 2. С. 489-501]. https://doi.org/10. 5800/GT-2018-9-2-0357.

Nikiforov V.M., Shkabarnya G.N., Kaplun V.B., Zhukovin A.Yu., Varentsov I.M., Pal'shin N.A., Cuong D.H., Chung N.N., Hung Z.K., 2018. Electrically Conductive Elements of Superdeep FluidFault Systems as Indicators of Seismically Active Zones at the Eastern Margin of the Eurasian Continent (Based on Magnetotelluric Sounding Data). Doklady Earth Sciences 480 (6), 730-738 (in Russian) [Никифоров В.М., Шкабарня Г.Н., Каплун В.Б., Жуковин А.Ю., Варенцов И.М., Пальшин Н.А., Куонг Д.Х., Чунг Н.Н., Хунг З.К. Электропроводящие элементы сверхглубинных флюидно-разломных систем как индикаторы сейсмически активных зон восточной окраины Евразийского континента (по данным магнитотеллурических зондирований) // Доклады АН. 2018. Т. 480. № 6. С. 730-738]. http://dx.doi.org/ $10.7868 /$ S0869565218180214.

Novikov I.S., Pospeeva E.V., 2017. Neotectonics of the Eastern Part of Gorny Altai According to Magnetotelluric Sounding Data. Russian Geology and Geophysics 58 (7), 959-971 (in Russian) [Новиков И.С., Поспеева Е.В. Неотектоника восточной части Горного Алтая по данным магнитотеллурического зондирования // Геология и геофизика. 2017. Т. 58. № 7. С. 959-971]. http://dx.doi. org/10.15372/GiG20170701.

Plotkin V.V., 2017. Technique of the Contribution Definition of the Hall Effect by Magnetotelluric Sounding. In: Subsoil Use. Mining. Directions and Technologies for Search, Exploration and Development of Mineral Deposits. Economics. Geoecology. Proceedings of International Scientific Conference. Vol. 2. Issue 3. Novosibirsk, 187-192 (in Russian) [Плоткин В.В. Методика определения вклада эффекта Холла при магнитотеллурическом зондировании // Недропользование. Горное дело. Направления и технологии поиска, разведки и разработки месторождений полезных ископаемых. Экономика. Геоэкология: Материалы Международной научной конференции. Новосибирск, 2017. Т. 2. № 3. С. 187-192].

Plotkin V.V., 2018. Manifestations of the Hall Effect by Results of the Polarizable Analysis of the Magnetotelluric Field. In: Subsoil Use. Mining. Directions and Technologies for Search, Exploration and Development of Mineral Deposits. Economics. Geoecology. Proceedings of International Scientific Conference. Vol. 4. Novosibirsk, 61-67 (in Russian) [Плоткин В.В. Проявления эффекта Холла по результатам поляризационного анализа магнитотеллурического поля // Недропользование. Горное дело. Направления и технологии поиска, разведки и разработки месторождений полезных ископаемых. Экономика. Геоэкология: Материалы Международной научной конференции. Новосибирск, 2018. Т. 4. С. 61-67].

Plotkin V.V., Mogilatov V.S., 2018. On the Role of the Hall Effect in Magnetotelluric Sounding. In: Problems of Geocosmos. Proceedings of 12th International School-Conference (October 8-12, 2018, Saint Petersburg, Peterhof). VVM
Publishing House, Saint Petersburg, 20-26 (in Russian) [Плоткин В.В., Могилатов В.С. О роли эффекта Холла при магнитотеллурическом зондировании // Проблемы геокосмоса: Материалы 12-й Международной школы-конференции (8-12 октября 2018 г., Санкт-Петербург, Петергоф). СПб.: Изд-во ВВМ, 2018. С. 20-26].

Plotkin V.V., Mogilatov V.S., Potapov V.V., 2019. Modifications of the Treffz Method for Estimating the Contribution of the Hall Effect in Magnetotelluric Sounding. Russian Geology and Geophysics 60 (3), 420-432 (in Russian) [Плоткин В.В., Могилатов В.С., Потапов В.В. Модификации метода Треффца для оценки вклада эффекта Холла при магнитотеллурическом зондировании // Геология и геофизика. 2019. T. 60. № 3. C. 420-432]. http://dx.doi.org/10.15372/ GiG2019017.

Pospeev A.V., 2012. The Velocity Structure of the Upper Mantle and Regional Deep Thermodynamics of the Baikal Rift Zone. Geodynamics \& Tectonophysics 3 (4), 377-383 (in Russian) [Поспеев B.A. Скоростная структура верхней мантии и региональная глубинная термодинамика Байкальской рифтовой зоны // Геодинамика и тектонофизика. 2012. Т. 3. № 4. С. 377-383]. https://doi.org/ 10.5800/GT-2012-3-4-0080.

Rybin A.K., Matyukov V.E., Batalev V.Yu., Bataleva E.A., 2019. Deep Geoelectric Structure of the Earth's Crust and Upper Mantle of the Pamir-Alai Zone. Russian Geology and Geophysics 60 (1), 127-139 (in Russian) [Рыбин А.К., Матюков В.Е., Баталев В.Ю., Баталева Е.А. Глубинная геоэлектрическая структура земной коры и верхней мантии Памиро-Алайской зоны // Геология и геофизика. 2019. T. 60. № 1. C. 127-139]. https://doi.org/10.15372/ GiG2019008.

Semenov V.Yu., 1985. Processing of Magnetotelluric Sounding Data. Nedra, Moscow, 133 p. (in Russian) [Семенов B.Ю. Обработка данных магнитотеллурического зондирования. М.: Недра, 1985. 133 с.].

Shuey R.T., 1975. Semiconducting Ore Minerals. Elsevier, New York, 287 p.

Singer B.S., 1992. Correction for Distortions of Magnetotelluric Fields: Limits of Validity of the Static Approach. Survey Geophysics 13, 309-340.

Wannamaker P.E., Hohmann G.W., Ward S.H., 1984. Magnetotelluric Responses of Three-Dimensional Bodies in Layered Earths. Geophysics 49 (9), 1517-1533. https://doi. org/10.1190/1.1441777.

Wunderman R.L., Wannamaker P.E., Young C.T., 2018. Architecture of the Hidden Penokean Terrane Suture and Midcontinent Rift System Overprint in Eastern Minnesota and Western Wisconsin from Magnetotelluric Profiling. Lithosphere 10 (2), 291-300. https://doi.org/10.1130/L716.1.

Yang B., Egbert G.D., Kelbert A., Meqbel N.M., 2015. ThreeDimensional Electrical Resistivity of the North-Central USA from Earthscope Long Period Magnetotelluric Data. Earth and Planetary Science Letters 422, 87-93. https://doi.org/ 10.1016/j.epsl.2015.04.006.

Zhdanov M.S., 1986. Electrical Prospecting. Nedra, Moscow, 320 р. (in Russian) [Жданов М.С. Электроразведка. М.: Недра, 1986. 320 с.]. 